\title{
PRODUTIVIDADE E QUALIDADE DE UVA, CV. ISABEL, EM DOIS SISTEMAS DE PRODUÇÃ O'
}

\author{
CESAR VALMOR ROMBALDI², MARIANO BERGAMASQUI ${ }^{3}$, LUCIANO LUCCHETTA ${ }^{4}$, MARCIO ZANUZO $^{4}$, \\ JORGE ADOLFO SILVA ${ }^{5}$
}

\begin{abstract}
RESUMO - Estudou-se a produtividade e a qualidade da uva, cv. Isabel, produzida em dois sistemas de produção, convencional e alternativo. O sistema convencional corresponde àquele empregado pela maioria dos produtores dessa cultivar, que consiste no controle de plantas concorrentes com o emprego de capinas e/ou herbicidas e no controle de doenças com fungicidas sintéticos orgânicos e não orgânicos. O sistema alternativo corresponde àquele onde se manteve a cobertura do solo e se inseriu a aveia como complemento, e o controle de doenças foi feito apenas com o emprego de calda bordalesa. Essas práticas foram realizadas desde 1998 e, nas safras 2001-2002 e 2002-2003, avaliaram-se a produtividade e a qualidade da uva produzida nos dois sistemas. Pelos resultados, observou-se que a produtividade e a qualidade da uva são mais afetadas pelas condições climáticas de safra do que pelo sistema de produção, indicando que o sistema de produção alternativo, onde se empregou menor número de pulverizações, não se usou herbicida, nem fungicida orgânico sintético, tem elevado potencial de adoção para essa cultivar.
\end{abstract}

Termos de indexação: Vitis, rendimento, maturação, cobertura do solo, videira.

\section{VINEYARD YIELD AND GRAPE QUALITY IN TWO DIFERENT CULTIVATION SYSTEMS}

\begin{abstract}
Vineyards of Vitis labrusca, cv. Isabel, were evaluated for yield and grape quality in two different cultivation systems: conventional and alternative. In the conventional cultivation system diseases and plant weeds are controlled by synthetic organic fungicides and herbicides, respectively. In the alternative system, diseases and plant weeds are controlled by cupric solution and oat mulching, respectively. The two production systems have been under general evaluation since 1998. In 2002 and 2003 the vineyards were evaluated in terms of yield and grape quality and none differences were found between cultivation systems despite the evident impact of climatic conditions. It suggests that the alternative system, where was used less aplications, it wasn't used herbicide and fungicidal organic synthetic, has a potential to be adopted for this cultivar.
\end{abstract}

Index terms: Vitis, yield, maturation, mulching, grapevine.

\section{INTRODUÇÃO}

A 'Isabel' é uma das principais cultivares de Vitis labrusca, espécie originária do Sul dos Estados Unidos e de onde foi difundida para outras regiões. Na década de 1850, despertou interesse dos viticultores europeus devido à resistência ao oídio, doença que naquela época causava enorme prejuízo à viticultura mundial (Grigoletti Jr. \& Sônego, 1993).

Foi introduzida no Rio Grande do Sul entre 1839 e 1842 por Thomas Maister, através da Ilha dos Marinheiros e, na atualidade, representa aproximadamente $40 \%$ de toda a uva produzida no RS. Os principais destinos da uva Isabel são a produção de vinho tinto comum, suco de uva, vinagre, geléias e comercializada como fruta in natura (Zanuz, 1991; Rizzon et al., 2000).

A expansão do cultivo com a cv. Isabel deu-se devido à sua fácil adaptação à variabilidade de condições edafoclimáticas, à elevada produtividade, à longevidade e à relativa rusticidade (Zanuz, 1991; Grigoletti Jr. \& Sônego, 1993).

O efeito do manejo da desbrota na produtividade e na qualidade da uva, da ação de bioestimulantes na produção da uva e na qualidade do mosto, e da maturação na qualidade da uva para vinho e suco já foi estudado (Passos \& Trintin, 1982; Miele, 1987; Zanuz, 1991).

$\mathrm{O}$ vinho produzido a partir da cv. Isabel apresenta aroma e gosto foxados. Mesmo assim, pelo hábito de consumo, associado às informações indicando os benefícios de pigmentos e taninos existentes nesse vinho, faz com que ainda seja o mais consumido no País e tenha grande potencial de expansão. Essa mesma lógica é válida para outros produtos derivados dessa cultivar, como vinagre, suco, geléia e a própria uva para consumo direto (Zanuz, 1991; Rizzon et al., 2000).

Porém, acompanhando as tendências mundiais, o consumidor nacional passou a valorizar cada vez mais aqueles alimentos produzidos em sistemas que estabeleçam um compromisso com a preservação do meio ambiente, da saúde do produtor e da estrutura de produção, sempre valorizando a interação consumidor-produtor, com produto final que atenda aos requisitos de segurança alimentar (Harker, 2003; Mars, 2003; Skuras \& Dimara, 2003). Daí a evolução dos sistemas de produção integrada, orgânica, biodinâmica, agroecológica e participativa de alimentos (Mars, 2003).

Neste contexto, embora se trate de uma cultivar relativamente rústica e produtiva, tem suscetibilidade à antracnose (Elsinoe ampelina) e míldio (Plasmopara viticola, L.). O emprego de fungicidas previne a ocorrência dessas fitopatias, mas, nas sucessivas safras, tem-se detectado indução de resistência e, em muitos casos, o incremento no número de pulverizações e de princípios ativos (Grigoletti Jr. \& Sônego, 1993). Por essas razões, os produtores têm buscado, junto às instituições de pesquisa e extensão, alternativas para evoluir em termos de sistema de produção, passando do estágio atual (convencional), onde se empregam capinas e/ou herbicidas e elevado número pulverizações com fungicidas sintéticos orgânicos e não orgânicos, para um sistema tecnologicamente mais "inteligente", onde se reduzam os insumos, o impacto ambiental e os riscos de intoxicação. Essa etapa tem sido denominada alternativa (Lapolli et al., 1995).

Pela importância socioeconômica que essa cultivar tem e pela necessidade de evoluir-se o sistema de produção, compararam-se dois sistemas de produção, convencional e alternativo, esse último sem a aplicação de herbicida e fungicidas orgânicos sintéticos, avaliando-se o potencial de produção e a qualidade da uva produzida.

\section{MATERIAL E MÉTODOS}

O trabalho foi realizado em cinco vinhedos, localizados na mesma propriedade, no município de Farroupilha-RS, nas safras de 2001-2002 e 2002-2003. Utilizou-se 1 hectare de cada vinhedo, todos da cv. Isabel, formados de pé-franco, implantados entre 1958 e 1962, conduzidos no sistema de latada, num espaçamento de 1,5 a 2,0m entre plantas e 3,0 a $3,5 \mathrm{~m}$ entre fileiras. $\mathrm{O}$ sistema de poda seca adotado foi o de cordão

\footnotetext{
${ }^{1}$ (Trabalho 126/2003). Recebido: 11/09/2003. Aceito para publicação: 02/04/2004. Financiado pela CAPES, FAPERGS e Emater.

${ }^{2} \mathrm{Ph}$. D., UFPEL/FAEM/DCTA, Cx. Postal 354, CEP: 96010900 - Pelotas - RS, cesarvrf@ufpel.tche.br

${ }^{3}$ M. Sc.,Rua Pinheiro Machado, 25, CEP: 95180000 - Farroupilha - RS.

${ }^{4}$ Mestrandos no Programa de Pós-Graduação em Ciência e Tecnologia Agroindustrial, UFPEL/FAEM/DCTA, Cx. Postal 354, CEP: 96010900 - Pelotas - RS.

${ }^{5}$ Prof. Adj. Dr., UFPEL/FAEM/DCTA, Cx. Postal 354, CEP: 96010900 - Pelotas - RS.
} 
esporonado. A partir da floração, realizou-se a poda verde, que consistiu na retirada de folhas e brotos sem frutificação.

Em 1996, em todos os vinhedos, foi realizada correção do solo com a aplicação de 12 t.ha ${ }^{-1}$ de calcário e 1,2 t.ha ${ }^{-1}$ de adubo NPK 5-20-20, atendendo às recomendações técnicas da análise de solo.

Até a safra de 1997, os vinhedos eram conduzidos pelo sistema convencional, ou seja, no mês de setembro, aplicava-se herbicida Glifosato, e iniciavam-se as pulverizações com Mancozeb, Metalaxil, Cymoxamil e Tiofanato Metílico, em intervalos de 6 a 8 dias, dependendo das condições climáticas. A partir da segunda quinzena de dezembro, realizavam-se aplicações de calda bordalesa, em número de 2 a 4 . A partir de 1998, 1ha de cada vinhedo foi mantido nesse sistema, sendo denominado convencional. O outro foi conduzido mantendo-se a cobertura do solo, associada à introdução de aveia-preta (Avena sativa, L.), no mês de março. No mês de setembro, efetuou-se uma roçada e não se aplicou herbicida. Para o controle de doenças, empregou-se somente calda bordalesa, na concentração de $1 \%$ a $2 \%$. Esse mesmo procedimento foi adotado nos anos seguintes.

$\mathrm{O}$ registro de dados, no que se refere à produtividade, foi estabelecido a partir de 1998, mas somente a partir de 2001-2002 passouse a avaliar a qualidade da uva nos dois sistemas. Por essa razão, serão apresentados apenas os dados referentes às duas últimas safras.

A produtividade foi determinada por pesagem, por ocasião da colheita, de toda a uva colhida por hectare, em cada um dos cinco vinhedos. Cada vinhedo constituiu-se numa repetição.

A avaliação da uva foi realizada, determinando-se, no mosto obtido após o desengace e esmagamento das bagas, a densidade, os sólidos solúveis totais, a acidez total titulável, o pH, a concentração de ácido tartárico, ácido málico, taninos e antocianinas. A metodologia seguiu exatamente os procedimentos descritos por Rizzon et al. (2000). Para cada variável analisada, realizaram-se 5 repetições. Para a comparação de médias, empregou-se o teste de Duncan a 5\%.

\section{RESULTADOS E DISCUSSÃO}

Ao analisarem-se os dados da Tabela 1, pode-se verificar que o efeito das condições de safra foi mais pronunciado do que o sistema de produção. Assim, por exemplo, em 2001-2002, a colheita pôde ser realizada entre 12 e 15-03, já que as condições climáticas permitiram, em função da não-ocorrência de chuvas e da baixa umidade relativa. Já, em 2002-2003, embora se tenha realizado um maior número de aplicações, houve necessidade de antecipar-se a colheita, em ambos os sistemas de produção. Isso se deu devido às condições climáticas adversas nesse ano agrícola, no qual foi detectada uma elevada incidência de chuvas no período de floração, enchimento do cacho e maturação. Isso resultou, também, numa menor qualidade da uva (Tabela 2). Mesmo com o aumento das pulverizações na safra 2002-2003, houve queda de produtividade, superior a 50\%, nos dois sistemas de produção. No sistema convencional realizaram-se 16 pulverizações, associando, em algumas delas, mais de um princípio ativo, e, mesmo assim, colheram-se apenas 13,25 toneladas por hectare. No sistema alternativo, a produtividade foi semelhante ao convencional, mesmo com o uso de apenas calda bordalesa.
Quando se comparam os sistemas de produção, verifica-se que eles não influenciaram na produtividade. Isso também foi verificado em anos anteriores (dados não apresentados), indicando que, para essa cultivar, e provavelmente para outras cultivares do grupo das Vitis labrusca, podem-se buscar alternativas, a curto prazo, para a substituição e subtração de insumos, reduzindo custos, impacto ambiental e riscos de contaminação. Na sequiência dos trabalhos, deverão ser realizadas análises complementares para detecção de resíduos dos princípios ativos empregados (Mancozeb, Metalaxil, Cymoxamil, Tiofanato Metílico e Cobre), além de micotoxinas e principais microrganismos de importância em termos de segurança alimentar. É importante destacar que, além da preocupação com segurança alimentar relacionada com fungicidas orgânicos sintéticos e herbicidas, o uso de produtos à base de cobre, nesse caso calda bordalesa, também preocupa pelas sucessivas aplicações e safras, que resulta em acúmulo no solo, elevando os riscos de toxicidade e contaminação dos lençóis freáticos.

As principais características da uva 'Isabel', nos dois sistemas de produção, estão apresentadas na Tabela 2. No sistema convencional, a densidade do mosto da uva produzida foi de $1,099 \mathrm{gmL}^{-1} \mathrm{em} \mathrm{2001-2002}$ e 1,061 $\mathrm{gmL}^{-1}$ em 2002-2003, e o conteúdo de sólidos solúveis totais foi de $18,2^{\circ}$ Brix em 2001-2002 e 16,5 ${ }^{\circ}$ Brix em 2002-2003. Já, no sistema alternativo, a densidade do mosto da uva produzida foi de $2,002 \mathrm{gmL}^{-1}$ em 2001-2002 e 1,051 gmL $\mathrm{gm}^{-1}$ em 2002-2003, e o conteúdo de sólidos solúveis totais foi de $18,5^{\circ}$ Brix em 2001-2002 e 15, $5^{\circ}$ Brix em 2002-2003.

O conteúdo de sólidos solúveis totais é o principal fator que afeta a densidade do mosto de uva (Amerine \& Ough, 1976), por essa razão foi observada maior densidade no mosto da safra 2001-2002, quando a uva evoluiu mais adequadamente à maturação. Os valores médios de sólidos solúveis totais observados podem ser considerados bons, já que a maioria das cultivares de Vitis labrusca geralmente apresentam menor potencial de produção de açúcar do que as cultivares de Vitis vinifera (Rizzon et al., 2000; Rizzon \& Miele, 2001). Quando se comparam as safras, também se verifica que, em 2002-2003, houve menor acúmulo de açúcares e menor densidade do mosto. Esse fato está relacionado com as condições climáticas nessa safra, quando, além da intensa ocorrência de chuvas no período da floração, houve elevada pluviosidade nos meses de janeiro a março $(580 \mathrm{~mm}$ no total do trimestre $)$, dificultando a maturação normal da uva.

A acidez total titulável também variou em função da safra, ou seja, em 2001-2002, quando as condições climáticas foram mais favoráveis à maturação, detectaram-se os menores valores de acidez, entre 68 e 74 meq. $L^{-1}$, já na safra 2002-2003, a acidez foi maior, entre 83 e 92 meq. $\mathrm{L}^{-1}$. Quanto ao pH, o valor mais elevado, de 3,44, foi detectado em 2001-2002 e o mais baixo de 3,24, em 2002-2003. Esses valores são elevados se comparados com os valores de $\mathrm{pH}$ de mostos de cultivares tintas de Vitis vinifera (Boulton, 1980; Rizzon et al., 1998; Rizzon et al., 2000; Rizzon \& Miele, 2001). O teor médio de ácido tartárico e málico também variou em função da safra, tendo apresentado maiores valores na safra 2002-2003 do que na de 2001-2002, dentro do mesmo sistema de produção.

No que tange aos taninos, os mostos apresentaram teores baixos, o que mostra o reduzido teor desses componentes nessa cultivar. As antocianinas, que são os principais componentes responsáveis pela

TABELA 1 - Número de pulverizações e produtividade de uva cv. Isabel, produzida nos sistemas de produção convencional e alternativo. FarroupilhaRS, 2003.

\begin{tabular}{|c|c|c|c|c|}
\hline \multirow[b]{2}{*}{ Variáveis } & \multicolumn{2}{|c|}{ Sistema Convencional } & \multicolumn{2}{|c|}{ Sistema alternativo } \\
\hline & $2001-2002$ & $2002-2003$ & $2001-2002$ & $2002-2003$ \\
\hline Data da colheita & 12 a $15-03$ & $1^{\circ}$ a $5-03$ & 12 a $15-03$ & $1^{\circ}$ a $5-03$ \\
\hline Número total de pulverizações & $9 c^{*}$ & $16 \mathrm{a}$ & $9 \mathrm{c}$ & $12 \mathrm{~b}$ \\
\hline Mancozeb & $5 b$ & $12 \mathrm{a}$ & $0 \mathrm{c}$ & $0 \mathrm{c}$ \\
\hline Metalaxil & $1 \mathrm{~b}$ & $2 \mathrm{a}$ & $0 \mathrm{c}$ & $0 \mathrm{c}$ \\
\hline Cymoxamil & $1 \mathrm{~b}$ & $2 \mathrm{a}$ & $0 \mathrm{c}$ & $0 \mathrm{c}$ \\
\hline Tiofanato metílico & $2 \mathrm{a}$ & $2 \mathrm{a}$ & $0 \mathrm{~b}$ & $\mathrm{Ob}$ \\
\hline Calda bordalesa & $2 \mathrm{~d}$ & $3 c$ & $9 b$ & $12 \mathrm{a}$ \\
\hline Produtividade $\left(\right.$ T.ha $\left.^{-1}\right)$ & $27,6 \mathrm{a}$ & $13,25 b$ & $28,1 \mathrm{a}$ & $12,36 b$ \\
\hline
\end{tabular}

*Letras distintas, na mesma linha, indicam que há diferença significativa entre as médias, pelo teste de Duncan, ao nível de 5\% de probabilidade. 
TABELA 2 - Características físico-químicas de mostos de uvas cv. Isabel, produzidas nos sistemas de produção convencional e alternativo. Farroupilha-RS, 2003.

\begin{tabular}{|c|c|c|c|c|}
\hline \multirow[b]{2}{*}{ Variáveis } & \multicolumn{2}{|c|}{ Sistema Convencional } & \multicolumn{2}{|c|}{ Sistema alternativo } \\
\hline & $2001-2002$ & $2002-2003$ & $2001-2002$ & $2002-2003$ \\
\hline Densidade a $20^{\circ} \mathrm{C}\left(\mathrm{g} \cdot \mathrm{mL}^{-1}\right)$ & $1,099 a^{*}$ & $1,061 \mathrm{~b}$ & $2,002 \mathrm{a}$ & $1,051 \mathrm{c}$ \\
\hline Sólidos solúveis totais $\left({ }^{\circ} \mathrm{Brix}\right)$ & $18,2 \mathrm{a}$ & $16,5 b$ & $18,5 \mathrm{a}$ & $15,5 \mathrm{c}$ \\
\hline Acidez total titulável (meq. $\mathrm{L}^{-1}$ ) & $74 \mathrm{c}$ & $83 b$ & $72 \mathrm{c}$ & $92 \mathrm{a}$ \\
\hline $\mathrm{pH}$ & $3,32 \mathrm{a}$ & $3,26 b$ & $3,44 a$ & $3,24 b$ \\
\hline Ácido tartárico $\left(\mathrm{g} . \mathrm{L}^{-1}\right)$ & $3,84 \mathrm{c}$ & $4,12 b$ & $3,24 d$ & $4,65 a$ \\
\hline Ácido málico $\left(\mathrm{g} . \mathrm{L}^{-1}\right)$ & $0,76 b$ & $1,02 \mathrm{a}$ & $0,74 b$ & $1,25 \mathrm{a}$ \\
\hline Taninos $\left(\mathrm{g} . \mathrm{L}^{-1}\right)$ & $0,46 a$ & $0,54 \mathrm{a}$ & $0,42 \mathrm{a}$ & $0,51 \mathrm{a}$ \\
\hline Antocianinas (mg. L $\left.{ }^{-1}\right)$ & $278 \mathrm{a}$ & $138 b$ & $284 a$ & $144 b$ \\
\hline
\end{tabular}

*Letras distintas, na mesma linha, indicam que há diferença significativa entre as médias, pelo teste de Duncan, ao nível de 5\% de probabilidade.

coloração da uva e seus derivados (Ribéreau-Gayon \& Stonestreet, 1965), foram afetadas pelas condições de cada safra, ou seja, quanto mais madura a uva estava, maior a concentração desse componente. Assim, em média, a uva da safra 2001-2002 continha duas vezes mais compostos antociânicos do que a da safra 2002-2003.

De maneira geral, na medida em que há evolução da maturação da uva, há tendência ao incremento do conteúdo de sólidos solúveis e redução da acidez. O aumento do conteúdo de sólidos solúveis se deve principalmente ao acúmulo de açúcares e pigmentos, à diminuição da acidez e à redução dos principais ácidos orgânicos da uva (Zanuz, 1991; Tamborra, 1992; Rizzon et al., 2000). O que foi observado nesse trabalho. No ano agrícola em que as condições climáticas foram melhores (20012002), pôde-se colher a uva mais tardiamente, o que permitiu a evolução da maturação, tendo-se obtido maior concentração de sólidos solúveis e pigmentos, menor acidez e menor concentração de ácidos tartárico e málico.

No que concerne aos sistemas de produção, não se observou efeito significativo das práticas de manejo sobre as principais variáveis de avaliação do mosto da uva cv. Isabel. Isso demonstra a possibilidade da redução e da substituição de insumos no sistema de produção, sem prejuízos em termos de produtividade (Tabela 1) e qualidade da uva (Tabela 2).

\section{CONCLUSÕES}

A produtividade e a qualidade da uva não foram afetadas pelos sistemas de produção, indicando que o sistema de produção alternativo, que empregou menor número de pulverizações, sem herbicida, nem fungicida orgânico sintético, pode ser adotado para essa cultivar.

\section{REFERÊNCIAS BIBLIOGRÁFICAS}

AMERINE, M.A.; OUGH, C.S. Análisis de vinos y mostos. Zaragoza: Acribia, 1976.158p.

BOULTON, R. The relationships between total acidity, titratable acidity and $\mathrm{pH}$ in wine. American Journal of Enology and Viticulture, Davis, v.31, n.1, p.76-80, 1980.

GRIGOLETTI Jr., A.; SÔNEGO, O.R. Principais doenças fúngicas da videira no Brasil. Bento Gonçalves. EMBRAPA-CNPUV, 1993. 36p.
(Circular Técnica, 17).

HARKER, F. R. Organic food claims cannot be substantiated through testing of samples intercepted in the marketplace: a horticulturalist's opinion. Food Quality and Preference, v.32, n.4, p.147-149, 2003.

LAPOLLI, J.M.; MELLO, L.M.R. de; TRARBACH, C. A Competitividade da vitivinicultura brasileira: análise setorial e programa de ação com destaque para o Rio Grande do Sul. Porto Alegre: BANRISUL/ EMBRAPA-CNPUV/SEBRAE, 1995.200p.

MARS, M. D. Agroecological innovation, increasing food production with participatory development. London: Norman Uphoff, 2003.306p.

MIELE, A. Teores de manganês e de cobre no mosto das uvas 'Isabel' e 'Concord'. Pesquisa Agropecuária Brasileira, Brasília, v. 22, n. 9/ 10, p. 897-901, 1987.

PASSOS, L.P.; TRINTIN, P.L. Influências da desbrota na produtividade e na qualidade da uva Isabel. Pesquisa Agropecuária Brasileira, Brasília, v. 17, n. 6, p. 859-864, 1982.

RIBÉREAU-GAYON, P.; STONESTREET, E. Le dosage des anthocyanes dans les vins rouges. Bulletin de la Societé Chimique de France, Paris, v.9, n.419, p.2649-2652, 1965.

RIZZON, L.A.; ZANUZ, M.C.; MIELE, A. Evolução da acidez durante a vinificação de uvas tintas de três regiões vitícolas do Rio Grande do Sul. Ciência Tecnologia de Alimentos, Campinas, v.18, n.2, p.179$183,1998$.

RIZZON, L. A.; MIELE, A. Avaliação da cv. Cabernet Franc para a elaboração de vinho tinto. Ciência Tecnologia de Alimentos, Campinas, v.21, n.2, p.249-255, 2001.

RIZZON, L. A.; MIELE, A.; MENEGUZZO, J. Avaliação da uva cv. Isabel para a elaboração de vinho tinto. Ciência Tecnologia de Alimentos, Campinas, v.20, n.1, p.115-121, 2000.

SKURAS, D.; DIMARA, E. Adoption of agricultural innovations as a two-stage partial observability process. Agricultural Economics, v.28, n.3, p.187-196, 2003.

TAMBORRA, P. L'acidità e gli equilibri di salificazione. L'Enotecnico, Milano, v.28, n.11, p.81-85, 1992.

ZANUZ, M.C. Efeito da maturação sobre a composição do mosto e qualidade do suco de uva. 1991. 177f. Dissertação (Mestrado em Fitotecnia) - Faculdade de Agronomia, Universidade Federal do Rio Grande do Sul. Porto Alegre, 1991 